\title{
Crecimiento, acumulación de biomasa seca y nutrientes en los frutos de café (Coffea arabica L.) variedad Pacas, Masatepe, Nicaragua
}

\section{Growth, and biomass and nutrient accumulation in coffee berries (Coffea arabica L.) pacas variety, Masatepe, Nicaragua}

\author{
Víctor Aguilar-Bustamante ${ }^{1}$, Rodolfo Munguía-Hernández ${ }^{1}$, Adán Chavarría-Rivera ${ }^{2}$, Nadia Calderón-Guido ${ }^{2}$ \\ ${ }^{1}$ Docentes Facultad de Agronomía, Universidad Nacional Agraria \\ ${ }^{2}$ Egresados Facultad de Agronomía, Universidad Nacional Agraria
}

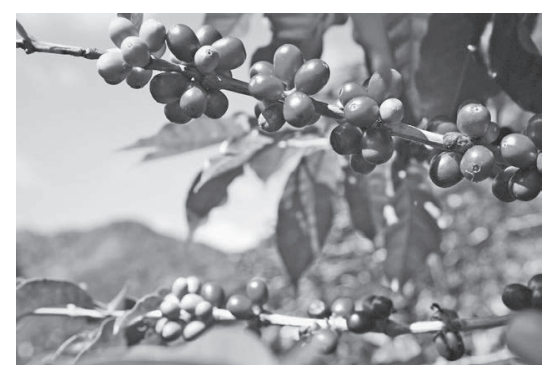

\section{RESUMEN}

De abril a diciembre de 2012, en un ensayo ya establecido se realizó un estudio en el cultivo de café variedad pacas, con el objetivo de determinar las curvas de crecimiento, acumulación de biomasa seca y acumulación de nutrientes $(\mathrm{N}, \mathrm{P}, \mathrm{K}, \mathrm{Ca}$ y $\mathrm{Mg}$ ) en los frutos de café uva. El ensayo fue establecido en el año 2000 en el Jardín Botánico hoy conocido como Centro Nacional de Educación y Cooperativismo (CENECCOP), Masatepe, Nicaragua. Se seleccionaron cuatro parcelas grandes con combinación de árboles de sombra maderables y de servicio y una parcela a pleno sol. Los tratamientos en estudio fueron a) Inga laurina y Simaruba glauca, b) Samanea saman y Tabebuia rosea, c) Samanea saman e Inga laurina, d) Tabebuia rosea y Simaruba glauca y e) una parcela a pleno sol. Las parcelas fueron distribuidas en un diseño de bloques completos al Azar (BCA). Las variables evaluadas en los frutos de café fueron diámetro polar, diámetro ecuatorial y peso seco. Los frutos se secaron en el horno a $70{ }^{\circ} \mathrm{C}$ y posteriormente se utilizó un molino Foss Tecator Cyclotec para obtener una muestra fina. De la muestra molida de los frutos, en el laboratorio de suelo y agua de la UNA se determinaron las concentraciones de N, P, K, Ca y Mg. Los datos fueron colectados cada 30 días. Para el análisis estadístico se utilizó SAS 9.1. Las curvas de crecimiento del fruto y la acumulación de peso seco se ajustaron a regresiones no lineales simples y dobles exponenciales. Se realizó una correlación entre el diámetro polar y diámetro ecuatorial de los frutos dando un coeficiente de determinación $\mathrm{r}$ de $98 \%$ $(\operatorname{Pr}>\mathrm{F}=0.0001)$. Para el ajuste de la curva de aumento de peso seco se utilizó el modelo Probit normal. El mayor crecimiento de los frutos se dio durante los primeros 90 días y la mayor acumulación de nutrientes se dio entre los 90 y 210 días. La combinación de árboles leguminosos presentó el diámetro polar y ecuatorial más alto. También la acumulación de biomasa seca fue mayor en el tratamiento con sombra de leguminosas.

Palabras clave: árboles de sombra, árboles de servicio, leguminosas,curva de crecimiento, elementos esenciales.

\section{ABSTRACT}

From April to December 2012, one study was conducted in a coffee plantation where pacas variety was planted, in order to determine the growth curves, dry weight and nutrient accumulation $(\mathrm{N}, \mathrm{P}, \mathrm{K}$, $\mathrm{Ca}$ and $\mathrm{Mg}$ ) in the coffee berries. The experiment was established in 2000 at Botanic Garden or the National Center for Cooperatives (CENECCOP) Masatepe, Nicaragua. This experiment consisted in four large plots with combination of shade trees and a fifth plot in an open sun was added. The treatment were a) Inga laurina and Simaruba glauca, b) Samanea saman and Tabebuia rosea, c) Samanea saman and Inga laurina d) Tabebuia rosea and Simaruba glauca and e) a coffee plot under open sun were sdded. A randomized Block Design was used. The measured variables in the coffee berries were polar diameter, equatorial diameter and dry weight. The coffee berries were oven dried at $70^{\circ} \mathrm{C}$ and milled to obtain a thin sample using a Foss Tecator Cyclotec mill. The thin sample was sent to UNA lab to measure the percentages of $\mathrm{N}, \mathrm{P}, \mathrm{K}, \mathrm{Ca}$ and $\mathrm{Mg}$. The data were collected every 30 days. The program used was Statistical Analysis System (SAS vs 9.1). A correlation between polar and ecuatorial diameter was run given one $\mathrm{r}$ of $98 \%(\mathrm{Pr}>\mathrm{F}=0.0001)$ The curves of growth and dry weight accumulation were adjusted using single and double exponential non-linear regressions. The dry weight accumulation curve was adjusted using a normal Probity model. The highest coffee berry growth occurred during the first 90 days and the nutrient accumulation was from 90 to 210 days. The dry weight accumulation was low during the first three months and increased slowly later on. The coffee berries under leguminous trees showed the highest growth. Olso the dry matter accumulation in the fruit was the highest in the leguminous trees.

Recibido: 27 de enero 2015 
$\mathrm{E}$ 1 café (Coffea arabica L.) es un cultivo que crece bajo sombra y crece favorablemente en zonas altas de Nicaragua. Es un sistema agroforestal que contribuye a la mitigación y adaptación de los efectos de los cambios climáticos debido a alta biodiversidad de árboles de sombra y frutales. Ayuda a la preservación de la fertilidad y la microbiología del suelo, así como a la retención y captura de agua. Este cultivo genera 300,000 empleos anuales tanto en labores de campo como en la cosecha y el beneficiado húmedo del café en pergamino. También genera empleo a empresas trilladoras de café, empresas torrefactoras y baristas en cafeterías. En términos de ingreso de divisas por exportaciones, en Nicaragua sigue siendo el principal motor, registrando al 31 de diciembre de 2012 más de 126,468 ha sembradas con una producción de 95,545 toneladas de café verde con un rendimiento promedio de $754.34 \mathrm{~kg} / \mathrm{ha}$ (FUNICA et al., 2013).

De la producción nacional en Nicaragua se destaca Jinotega, como el mayor productor de café oro con un $45 \%$, Matagalpa con 34\%, Las Segovias con 15\%, Carazo con $2.8 \%$, Boaco con $1.4 \%$, Managua con $1.7 \%$, y Chinandega con $0.1 \%$. En el pacífico, la mayor parte de los cafetales está en la zona llamada meseta de Carazo, que incluye parte de los departamentos de Masaya, Granada y Managua. Las plantaciones del pacífico se encuentran por debajo de los $600 \mathrm{msnm}$ y la zona con mejores condiciones es la denominada Triángulo de oro entre Diriamba, Jinotepe y San Marcos. Son suelos planos y profundos, temperatura de $26{ }^{\circ} \mathrm{C}$ y precipitaciones de hasta $1400 \mathrm{~mm}$ (CATIE, 2000).

En la zona del Pacífico, en el mes de abril de cada año se presentan lluvias erráticas conocidas como jocoteros o cabañuelas. Estas lluvias, estimulan la floración temprana conocidas como floraciones locas que al no continuar las lluvias las plantas no logran retener los frutos. En la zona cafetalera de Carazo donde existe una época seca muy marcada de noviembre a abril de cada año, se espera que al inicio de las lluvias en mayo se presente una floración fuerte y uniforme. Carazo como una zona baja y seca no escapa de los efectos de estas precipitaciones donde se pudieron observar en el año 2012, siete floraciones, producto de las lluvias caídas durante los meses de marzo, abril y mayo.

A principios de año 2012, las siete floraciones ocurridas fueron producto de las lluvias caídas el 3, 18, y 21 de enero, el 9 de febrero, 9 de marzo, 21 de abril y 21 de mayo. Hasta mayo se habían registrado $170.8 \mathrm{~mm}$. El 21 de abril se registraron $21 \mathrm{~mm}$ dando como producto la floración principal estimada en un 70\% del ensayo de sistemas de café establecido en el CENECOOP (Centro Nacional de Educación para el Cooperativismo) anteriormente conocido como Jardín Botánico, por lo que esta floración y fructificación se utilizó para realizar este estudio que se presentan en este artículo científico.
De los frutos, producto de la floración estimulada por la lluvia del 21 de abril se seleccionaron bandolas productivas con los objetivos de conocer el comportamiento del crecimiento y acumulación de peso seco y la acumulación de nutrientes de los frutos de café bajo combinaciones de especies de árboles de sombra y a plena exposición solar con la aplicación de fertilizante convencional moderado.

\section{MATERIALES Y MÉTODOS}

Ubicación del experimento. Este estudio fue realizado de abril a diciembre del año 2012, en una plantación de café variedad pacas de 11 años de establecida en el centro experimental Jardín botánico, hoy CENECOOP (Haggar y Staver, 2003). El centro está localizado a $11^{0} 54^{\prime}$ latitud norte y $86^{\circ}$ 09' longitud oeste. Tiene una altitud de $455 \mathrm{msnm}$. La temperatura promedio anual es de $26^{\circ} \mathrm{C}$. Es una zona baja y seca con suelos profundos y fértiles. Precipitación promedio en los últimos 10 años de $1400 \mathrm{~mm}$. La precipitación acumulada registrada en el año de estudio 2012 fue de $970.7 \mathrm{~mm}$.

Diseño metodológico. Para el estudio se tomaron cuatro parcelas bajo sombra y una a plena exposición solar: a) Inga laurina-Samanea saman (ILSS), b) Tabebuia rosea-Simarouba glauca (TRSG), c) Tabebuia rosea-Samanea saman (TRSS), d) Inga laurina-Simarouba glauca (ILSG) y e) a pleno sol. El tamaño de cada parcela fue de $48 \mathrm{~m}$ de ancho por $60 \mathrm{~m}$ de largo con un área de $2880 \mathrm{~m}^{2}$. La densidad de siembra fue de 4000 plantas por hectárea a una distancia entre surcos de 2 metros y entre planta y planta de 1.25 metros. Los árboles de sombra estaban establecidos a una distancia de 6.5 metros sobre el surco y 8 metros entre surcos para una densidad de 192 árboles por hectárea.

Metodología de muestreo. Para medir el crecimiento del fruto de café uva, el aumento de la biomasa y la acumulación de nutrientes se seleccionaron ocho plantas de café de la parcela útil, marcándose una bandola del tercio medio de la planta en el área más productiva de la misma. Se hicieron ocho mediciones donde se tomaron los frutos de una bandola por tratamiento desde mayo a diciembre. La cinta plástica se ubicó en la base de la bandola y las variables de crecimiento, acumulación de biomasa y acumulación de nutrientes se realizaron cada 30 días en 10 frutos de la bandola desde mayo a diciembre 2012. Las variables evaluadas fueron: Diámetro polar ( $\mathrm{mm})$, diámetro ecuatorial $(\mathrm{mm})$, peso seco de cada fruto $(\mathrm{g})$, contenido en porcentaje de nitrógeno, fósforo, potasio, calcio y magnesio.

Contenido de nutrientes (\%). Para determinar el contenido de los nutrientes las muestras de frutos secos se prepararon en un molino FOSS Cyclotec ${ }^{T M} 1093$ posteriormente enviadas al Laboratorio de Suelos y Agua (LABSA) de la UNA. El análisis se hizo en los siguientes elementos: Nitrógeno, 
Fosforo, Potasio, Calcio y Magnesio; se utilizó el método de la digestión sulfoselénica (Digestión húmeda) con el que se obtuvo el porcentaje de nutrientes concentrado en los frutos de café durante su crecimiento.

Análisis de datos. Se realizó análisis de correlación para determinar el comportamiento del diámetro polar y el diámetro ecuatorial. Para predecir o simular el diámetro polar, el diámetro ecuatorial y la acumulación de peso seco, se compararon los modelos de regresión simple lineal, modelo simple exponencial, modelo doble exponencial, modelo asimptótico y modelo probit normal. Para seleccionar el mejor modelo de ajuste se escogió el valor más alto de la $\mathrm{r}^{2}$ y el menor valor del cuadrado medio del error (CME).

Manejo agronómico. Las plantas de café fueron establecidas en el año 2000. La fertilización consistió en $181 \mathrm{~kg} / \mathrm{ha}$ de completo 12-30-10 en julio y agosto y $45 \mathrm{~kg} / \mathrm{ha}$ de urea octubre. Se fertilizó con cobre, zinc y boro en mayo y julio a razón de $2.5 \mathrm{~g} / \mathrm{L}$ de agua. Las malezas fueron controladas con chapeas y uso del glifosato $35.6 \mathrm{~L} \mathrm{a} 3 \mathrm{cc} /$ cada tres meses. Se realizaron labores de deshije. El ataque de roya (Hemileia vastatrix) fue severo ya que no se aplicaron productos para su manejo. Los frutos afectados por broca (Hypotenemus hampei) no pasó del 5\% a pesar de una aplicación de Thiodán 35 EC $3 \mathrm{cc} / \mathrm{L}$. No se realizó regulación de sombra durante el estudio.

\section{RESULTADOS Y DISCUSIÓN}

Variables de crecimiento de frutos de café uva. Los frutos de café presentaron un rápido incremento de tamaño hasta los 90 días después de la floración (ddf) momento en que alcanzaron en promedio un $93 \%$ de diámetro polar y $75 \%$ de diámetro ecuatorial, para luego estabilizarse hasta la etapa de maduración donde vuelve a presentar incrementos de tamaño.

En cuanto al diámetro polar, la combinación de sombra ILSS (guaba y genízaro) presentó los mayores valores hasta los 90 ddf, sin embargo en ese momento no mostró diferencias significativas $(\mathrm{Pr}=0.6017)$ con los demás tratamientos, mientras que café a pleno sol mostró los menores diámetros. Para el momento de cosecha, los resultados mostraron que hay diferencia significativas $(\mathrm{Pr}=0.0054)$, la combinación de TRSS (roble y genízaro) fue superior a los demás tratamientos, los frutos presentaron un diámetro de $15.1 \mathrm{~mm}$ y el tratamiento TRSG (roble y acetuno) presentó el valor más bajo con $13.86 \mathrm{~mm}$.
Los mayores valores de diámetro ecuatorial los presentó TRSS (roble y genízaro). A los 90 ddf momento en que los frutos alcanzaron la mayor parte de su tamaño máximo, no se presentó diferencias significativas $(\mathrm{Pr}=0.5230)$ entre los tratamientos. Los frutos en la sombra con TRSS fueron estadísticamente superior $(\mathrm{Pr}=0.0101)$ a los demás tratamientos con un diámetro de $13.62 \mathrm{~mm}$ y TRSG presentó el valor más bajo $12.71 \mathrm{~mm}$. Cabe señalar que el café a pleno sol los frutos se posicionaron en la misma categoría estadística que las combinaciones que presentaron superioridad, es decir no existió diferencia entre las combinaciones de sombra ILSS, TRSS, y a pleno sol (figura 2).

Acumulación de peso seco en frutos de café. El aumento de peso de los frutos (figura 3) mostró una curva sigmoide simple. A los 90 ddf en promedio para todos los tratamientos los frutos llegaron a pesar un $30 \%$ de su peso máximo, este comportamiento sugiere que en la fase inicial de desarrollo una mayor parte del fruto está constituida por agua y en los próximos meses de desarrollo gana peso, a medida que avanza la maduración y ocurre el llenado, así como también debido a los cambios ocurridos en la pulpa (Sadeghian et al., 2013). 


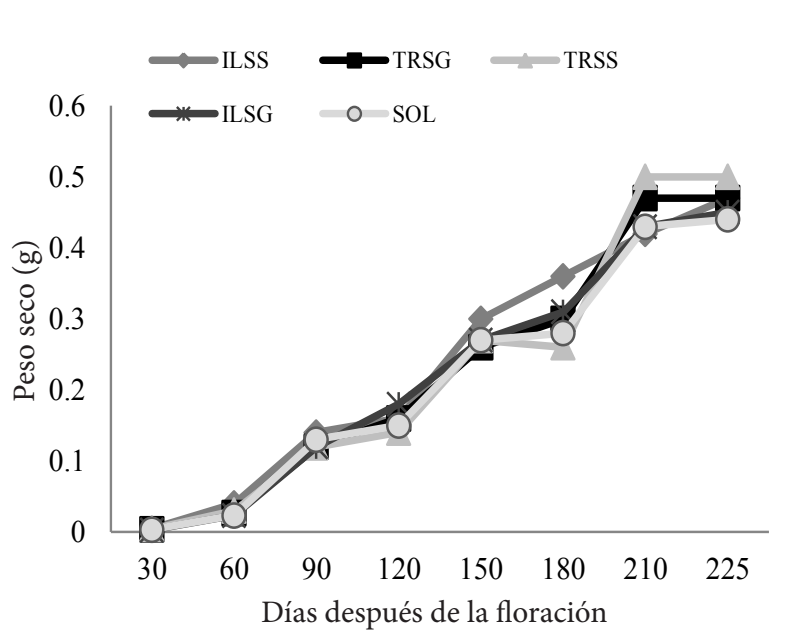

Figura 3. Curvas de acumulación de peso seco bajo combinaciones de sombra y a pleno sol, Masatepe, Nicaragua.

Entre las combinaciones de sombra, ILSS mostró mayor peso en los frutos desde su primer estado inicial de desarrollo hasta los 180 ddf coincidiendo estos días con la etapa de maduración de los mismos. También los frutos con ILSS presentaron una acumulación de biomasa progresivamente constante durante todo el periodo de desarrollo de los 90-180 ddf. De los 180 a los 210 ddf las combinaciones de sombra TRSG, TRSS, ILSG y café a pleno sol, mostraron un marcado incremento en el peso de los frutos.

Al momento de la cosecha ILSS y TRSS alcanzaron el mayor valor de peso con $0.47 \mathrm{~g}$ y el menor lo presentó los frutos de café a pleno sol con $0.44 \mathrm{~g}$. Se debe destacar que los frutos bajo pleno sol presentaron bajo peso en comparación con algunas combinaciones de sombra, sin embargo, no fueron los menores valores en el desarrollo del fruto, únicamente en dos momentos determinados, estos frutos mostraron el menor peso a los 60 y 225 ddf con 0.023 g y $0.44 \mathrm{~g}$ respectivamente.

Correlación entre el diámetro polar y el diámetro ecuatorial. Se realizó una correlacion de Pearson para conocer la relacion lineal entre el diámetro polar y el diamtro ecuatorial, la cual mostró una correlacion postiva $(\mathrm{r}=0.9800)$ lo que indica una dependencia en un $98 \%$ entre el diámetro polar y el diámetro ecuatorial. Estas dos variables tienen una relación directa ya que a medida que una aumenta la otra lo hace en proporción constante. Entre los 90 ddf hasta los 210 ddf existe una separacion un poco mas marcada en la cual el díametro ecuatorial tiende a crecer menos, manteniendo la misma tendencia (figura 4).

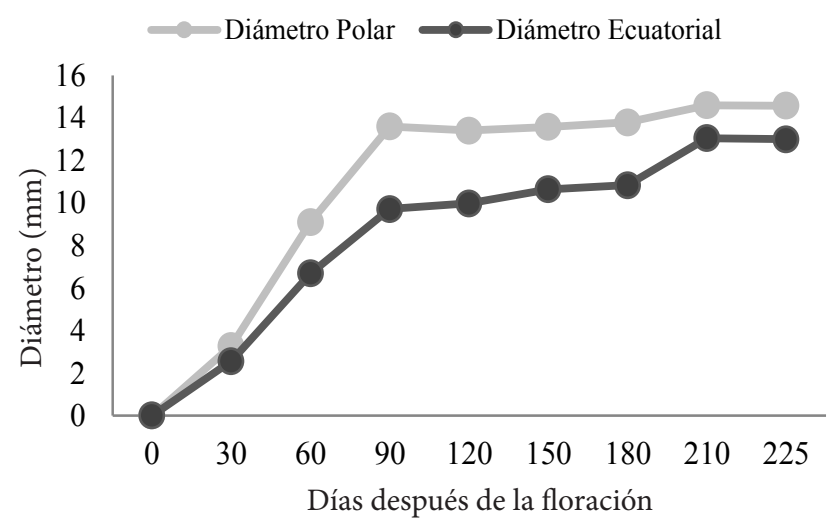

Figura 4. Correlación entre el diámetro polar y diámetro ecuatorial de los frutos de café, Masatepe, Nicaragua.

Crecimiento exponencial del diámetro polar y diámetro ecuatorial. El aumento del diámetro polar de los frutos en los primeros 90 ddf experimentaron un crecimiento rápido y luego se estabilizaron hasta la maduración del fruto. Este comportamiento no obedece a una regresión lineal. En este caso se debe probar el mejor ajuste de curvas usando una función no lineal, utilizando conceptos de inferencia estadística.

De igual forma, la tendencia del diámetro polar fue igual que el diámetro ecuatorial donde se presentó un crecimiento rápido en los primeros 90 días aunque un poco más lento y con una tendencia más uniforme. Meter et al., (1996) recomienda considerar en este caso usar modelos de regresión no lineal para estimar la curva de crecimiento en el tiempo como el modelo simple exponencial, modelo doble exponencial, modelo asimptótico, modelo de regresión logístico y modelo Probit.

$\mathrm{Al}$ correr los modelos arriba mencionados el modelo no lineal simple exponencial fue el modelo en el que los datos observados se ajustaron mejor a los datos esperados con $\mathrm{r}^{2}=$ 0.9913 para el diámetro polar y $\mathrm{r}^{2}=0.9937$ para el diámetro ecuatorial (figura 5).

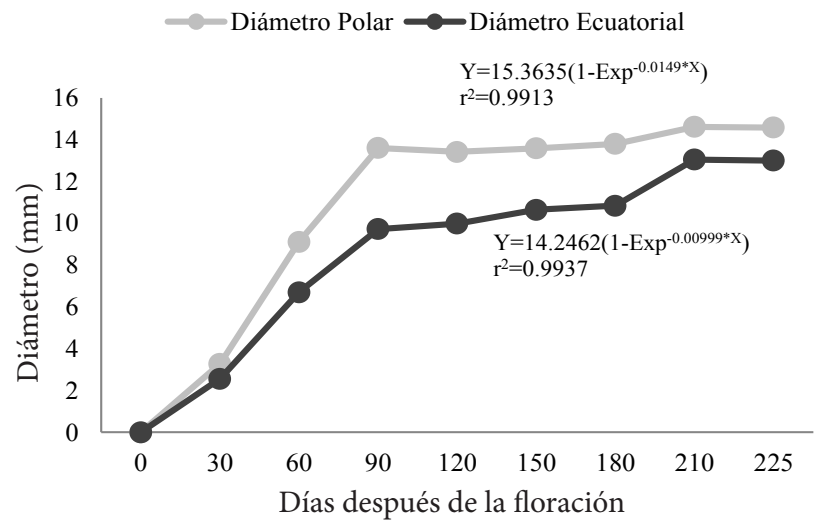

Figura 5. Crecimiento exponencial de los diámetros usando el método de regresión no lineal propuesto por Marquardt $Y=b o^{*}\left(1-\operatorname{Exp}^{-b 1 * X}\right)$. 
Comportamiento del peso seco de frutos de café, ajustado al modelo Probit. En la curva de aumento de peso seco, puede observarse una curvatura hacia abajo del eje X. primero experimenta un crecimiento lento hasta los 60 días, luego crece rápidamente hasta los 210 días y vuelve a estabilizarse hasta los 225 días. El modelo que mejor se ajustó a la distribución de los datos de la acumulación de peso seco en el tiempo fue el modelo Probit que es el inverso de los valores acumulativos de la distribución normal (figura 6). A los 90 días hay una acumulación de peso del 26\%, a los 180 días un $65 \%$ y finalmente a los 210 días una acumulación de $98 \%$

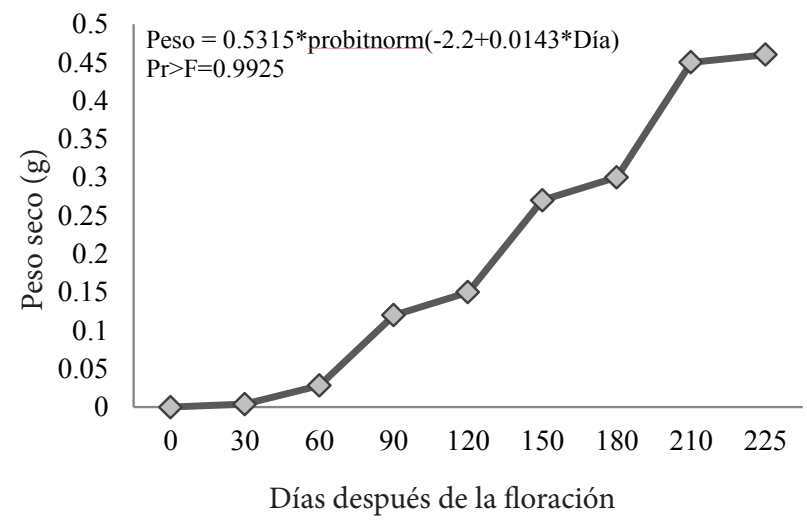

Figura 6. Comportamiento del peso seco $(\mathrm{g})$ de los frutos de café desde la floración hasta la maduración según modelo probit $\mathrm{Y}=\mathrm{a}$ *probnorm $(\mathrm{a}+\mathrm{b}$ *días $)$.

Concentración de nutrientes en los frutos de café bajo cuatro combinaciones de sombra y a pleno sol.

Las concentraciones de nutrientes en los frutos de café bajo cuatro combinaciones de sombra y a pleno sol mostraron en determinado momento del desarrollo una reducción en su contenido, en los nutrientes N, K y Ca se da a los 60 ddf y en P y Mg se da a los 90 ddf.

La mayor concentración de N, la presentaron los frutos de café bajo la combinación TRSS a lo largo de todo su desarrollo, sin embargo cabe señalar que ILSS presentó en dos momentos definidos valores más altos en la concentración de este nutriente a los 120 ddf con $2.15 \%$ y a los $150 \mathrm{ddf}$ con $1.84 \%$. Para el momento de la cosecha los tratamientos TRSS, ILSG y a plena exposición solar mostraron la mayor concentración con $2.05 \%$ y TRSG el menor porcentaje con 1.58\%. Russo y Botero (1996), señalan que los árboles fijadores de nitrógeno incrementan el nivel de nitrógeno en el suelo debido a su capacidad de fijarlo de la atmósfera, y por medio del aporte de materia orgánica. Además, sus raíces pueden absorber nutrientes de capas profundas del suelo y movilizarlos a la superficie, haciéndolos disponibles para el cultivo. En algunos casos, pueden incrementar la disponibilidad de fósforo (simbiosis con micorrizas), calcio, potasio y magnesio.
A los 90 ddf los sistemas ILSG con $0.43 \%$ presentó los mayores valores de concentración de $\mathrm{P}$ y Pleno sol $0.13 \%$ presentó los menores (figura 7). A los 150 ddf se observó que el tratamiento ILSG con $0.02 \%$, presentó un descenso considerable en comparación a los demás tratamientos que también presentaron una disminución de $\mathrm{P}$, sin embargo este mismo tratamiento a los 180 ddf logro estabilizarse entre los otros tratamientos llegando a tener una concentración de $0.12 \%$, valor cercano e incluso mayor que las demás combinaciones de sombra.

En el caso del K, mostró un aumento a los 90 ddf donde TRSS con $3.61 \%$, se presentó como el tratamiento de mayor concentración y Pleno sol $2.88 \%$ con el menor valor, después de este comportamiento todos los tratamientos fueron descendiendo de manera lenta, a los 150 ddf se observó que Pleno sol con $3.16 \%$ tuvo un incremento mientras ILSG mostró una reducción bien marcada con $0.97 \%$. Al momento de la cosecha ILSG mostró la mayor concentración de $\mathrm{K}$ con $1.99 \%$ y ILSS presento la menor concentración con $1.33 \%$.

La concentración de Ca presentó un comportamiento más constante, sin embargo se logra observar que a los 90 ddf ILSG $0.58 \%$ presentó un aumento a diferencia de los demás tratamientos que siguieron bajando su concentración. Para el momento de cosecha TRSS con $0.31 \%$ es el tratamiento con mayor concentración de Ca en los frutos y TRSG con $0.14 \%$ es el de menor concentración (figura 7).

Forrester et al.,(2006), señala que los estudios de producción y descomposición de materia orgánica de especies que crecen asociadas, fijadoras de $\mathrm{N}$ con especies no leguminosas, han sido escasos pero con resultados muy prometedores en cuanto a la calidad y pertinencia en la liberación e incorporación de nutrimentos al suelo y Hättenschwiler et $a l .,(2005)$ indica que ocurre un mayor flujo de nutrimentos causados principalmente por las diferencias en la composición química y propiedades físicas de la hojarasca originadas por las distintas especies.

La concentración del $\mathrm{Mg}$ mostró una gradual reducción desde los 90 ddf en todas las combinaciones de sombra, cabe señalar que los frutos de café a pleno sol mostraron mayor concentración de Mg hasta los 150 ddf. A la cosecha de los frutos TRSS con $0.53 \%$ mostró la mayor concentración de dicho nutriente e ILSS con $0.16 \%$ se presentó como el menor valor de concentración.

La producción y descomposición (humificación y mineralización) de la materia orgánica hace la conexión entre el componente biótico y abiótico del sistema (Heuveldop et al., 1988). Lo anterior, tiene una importancia potencial para el reciclaje de $\mathrm{N}, \mathrm{P}, \mathrm{K}, \mathrm{Ca}$ y $\mathrm{Mg}$, en donde los contenidos de nutrientes frecuentemente exceden las entradas de fertilizantes inorgánicos incluso cuando él último es aplicado a niveles altos para café (Beer, 1988). 


\section{PRODUCGIOONDEPLANZTAS}

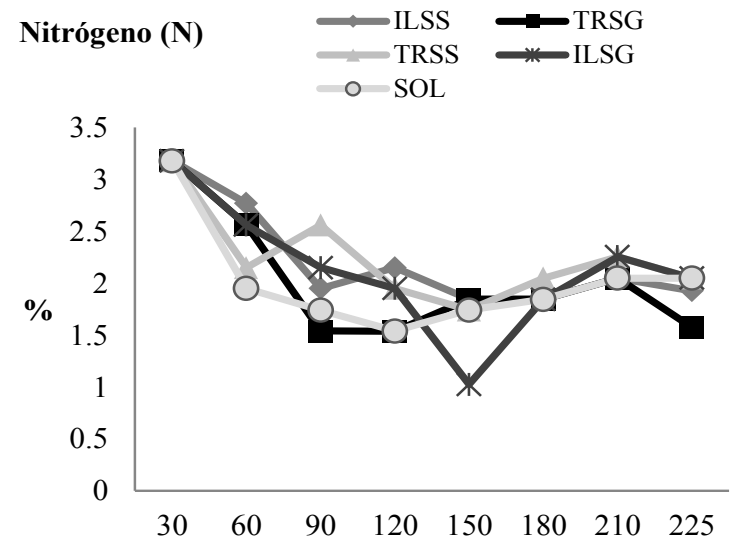

Fósforo (P)

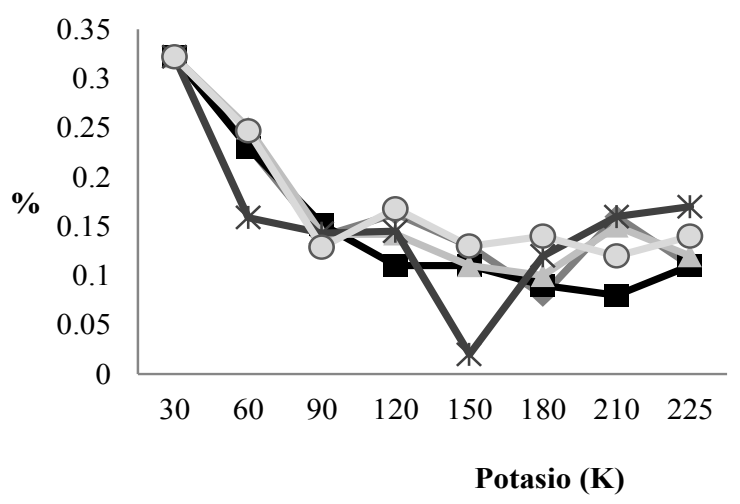

Calcio (Ca)

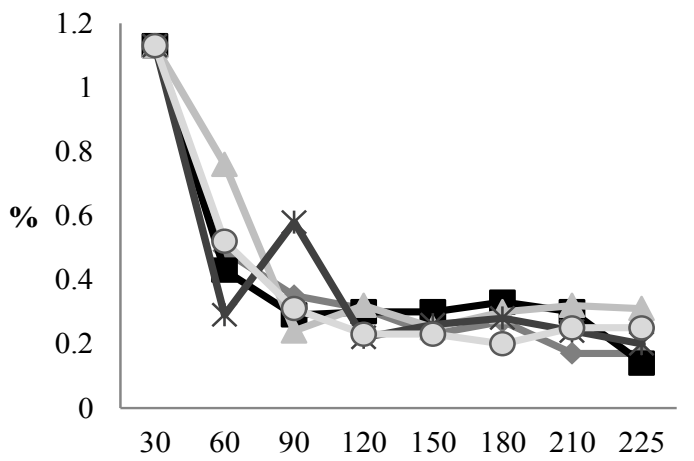

Magnesio (Mg)

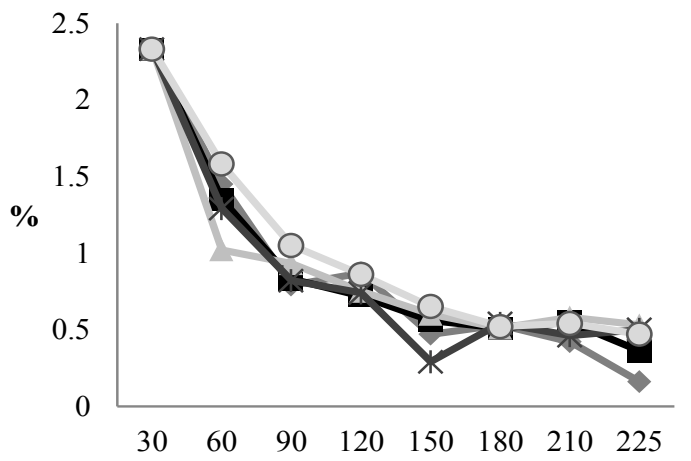

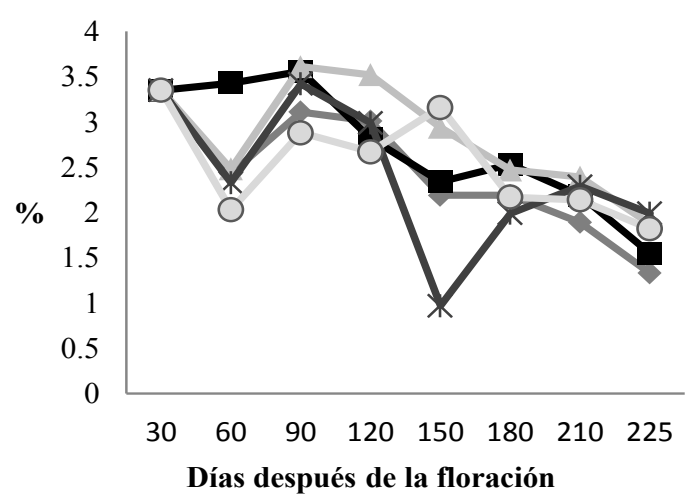

Figura 7. Concentración de nutrientes en los frutos de café uva bajo cuatro combinaciones de sombra y a pleno sol. Masatepe, Nicaragua.

\section{CONCLUSIONES}

Los frutos bajo la sombra de árboles de leguminosas y la combinación de leguminosas y no leguminosas tuvieron mayor diámetro polar y ecuatorial, a pleno sol presentaron valores intermedios y los frutos con menor crecimiento se obtuvieron con sombra de árboles no leguminosas.

Los frutos bajo sombra de leguminosas y combinaciones de leguminosas y no leguminosas presentaron el mayor peso seco. El menor peso seco de los frutos se presentó en los granos de café a pleno sol.

La concentración inicial de los nutrientes en los frutos de café en orden de mayor a menor fue K $(3.5 \%)$, N $(3.2 \%)$,
$\mathrm{Mg}(2.5 \%), \mathrm{Ca}(1.2 \%)$ y $\mathrm{P}(0.3 \%)$. A la cosecha la demanda de nutrientes descendió a un $17 \%$ para $\mathrm{Ca}, 18 \%$ para $\mathrm{Mg}, 37.5 \%$ para $\mathrm{P}, 45 \%$ para $\mathrm{K}$ y $58.5 \%$ para $\mathrm{N}$.

La mayor acumulación de nutrientes ocurrió entre los 90 y 210 días. Los frutos con leguminosas acumularon mayor cantidad de N, K, Mg Y P, con no leguminosas mayor cantidad de Ca.

Los modelos matemáticos que más se ajustaron, al crecimiento de los frutos de café fue el modelo de regresión simple exponencial y para la acumulación de peso seco al modelo Probit normal. 


\section{PRODOCCIOONDEPLANUEAS}

\section{REFERENCIAS BIBLIOGRÁFICAS}

Beer, J. 1988. Litter production and nutrient cycling in coffee (Coffea arabica) or cacao (Theobrama cacao) plantations with shade trees. Agroforestry Systems, 7:103-114.

CATIE (Centro Agronómico Tropical de Investigación y Enseñanza) 2000. Cuadernos prácticos de capacitación para productores y técnicos de café. $30 \mathrm{p}$.

Forrester D, I; Bauhus, J; Cowie A, L; Vanclay, J. 2006. Mixed-species plantations: of Eucalyptus globules and Acacia mearnsii with nitrogen-fixing trees: a review. For. Ecol. Managem. 233:211-230.

FUNICA (Fundación para el Desarrollo Tecnológico Agropecuario y Forestal de Nicaragua); UPOLI (Universidad Politécnica de Nicaragua); ICIDRI (Instituto de Capacitación e Investigación en Desarrollo Rural Integrado);UNA (Universidad Nacional Agraria); ESETECA (Empresa de Servicios Tecnológicos Agropecuarios S.A). 2013. Validación de alternativas no sintéticas en el manejo integrado de plagas del café en el pacifico sur de Nicaragua.11 p.

Haggar J; de Melo, E; Staver, C. 2003. Sostenibilidad y sinergismo en café agroforestal. Ensayo de sistemas para la producción de café. Programa Regional CATIE/MIP - AF (NORAD). 102 p.

Hättenschwiler, S; Tiunov A, V; Scheu, S. 2005. Biodiversity and litter decomposition in terrestrial ecosystems. Annu. Rev. Ecol. Evol. Syst. 36:191-218.

Heuveldop, J; Fassbender, HW; Alpizar, L; Enriquez, G; Folster, H. 1988. Modelling agroforestry systems ofcacao (Theobrama cacao) with laurel (Cordia alliodora) andporo (Erythrina poeppigiana) in Costa Rica. II. Cacao and Wood production, litterproduction and decomposition. AgroforestrySystems 6: $37-48$.

Russo, R.O; Botero.R. 1996. Nitrogen fixing trees for animal production on acid soils. In: Powell, M.H. (ed.). Nitrogen fixing trees for acid soils: a field manual. Nitrogen Fixing Tree Association (NFTA). Morrilton, Arkansas. p 31-39.

Sadeghian K, S; Mejía M, B; González O, H. 2013a. Acumulación de Nitrógeno, Fosforo y Potasio en los frutos de café. Avances Técnicos Cenicafe. 409: 1-8.

2013b. Acumulación de calcio, magnesio y azufre en los frutos de café. Avances técnicos Cenicafe. 430: 1-8. 